\title{
Revisiting the eccentricities of hot Jupiters
}

\section{Nawal Husnoo ${ }^{1}$, Frédéric Pont ${ }^{1}$, Tsevi Mazeh ${ }^{2}$, Daniel Fabrycky ${ }^{3}$, Guillaume Hébrard ${ }^{4,5}$ and Claire Moutou ${ }^{6}$}

\author{
${ }^{1}$ School of Physics, University of Exeter, Exeter, EX4 4QL, UK \\ email: nawal@astro.ex.ac.uk \\ ${ }^{2}$ School of Physics and Astronomy, Tel Aviv University, Tel Aviv 69978, Israel \\ ${ }^{3}$ Harvard-Smithsonian Centre for Astrophysics, Garden Street, Cambridge MA \\ ${ }^{4}$ Institut d'Astrophysique de Paris, UMR7095 CNRS, Université Pierre \& Marie Curie, 98bis \\ boulevard Arago, 75014 Paris, France \\ ${ }^{5}$ Observatoire de Haute-Provence, CNRS/OAMP, 04870 Saint-Michel-l'Observatoire, France \\ ${ }^{6}$ Laboratoire d'Astrophysique de Marseille, Université de Provence, CNRS(UMR 6110), 38 rue \\ Frédéric Joliot Curie, 13388 \& Marseille cedex 13,France
}

\begin{abstract}
Most short period transiting exoplanets have circular orbits, as expected from an estimation of the circularisation timescale using classical tidal theory. Interestingly, a small number of short period transiting exoplanets seem to have orbits with a small eccentricity. Such systems are valuable as they may indicate that some key physics is missing from formation and evolution models. We have analysed the results of a campaign of radial velocity measurements of known transiting planets with the SOPHIE and HARPS spectrographs using Bayesian methods and obtained new constraints on the orbital elements of 12 known transiting exoplanets. We also reanalysed the radial velocity data for another 42 transiting systems and show that some of the eccentric orbits reported in the Literature are compatible with a circular orbit. As a result, we show that the systems with circular and eccentric orbits are clearly separated on a plot of the planetary mass versus orbital period. We also show that planets following the trend where heavier hot Jupiters have shorter orbital periods (the "mass-period relation" of hot Jupiters), also tend to have circular orbits, with no confirmed exception to this rule so far.
\end{abstract}

Keywords. planetary systems

\section{Introduction}

Transiting planets are the only exoplanets for which we can precisely measure the orbital and physical parameters relevant in constraining planet formation and migration models. Today, over one hundred transiting planets are known, most of which orbit their parent stars at a distance smaller than 0.1 AU. These close-in planets are expected to experience strong tidal forces, and their orbits are expected to circularise early in their lifetimes (Lin, Bodenheimer \& Richardson 1996).

It is therefore surprising that a significant number of transiting systems with very short periods, such as WASP-12 and WASP-18 (see Figure 1), appear to have small but measurable eccentricities at the few percent level from radial velocity data and transit photometry. For example, WASP-12b was reported by Hebb et al. (2009) to have an eccentricity of $e=0.049 \pm 0.015$, while orbiting at only 3.1 stellar radii. WASP-18 was reported by Hellier et al. (2009) to have $e=0.0092 \pm 0.0028$ and this was refined by Triaud et al. (2010) to $e=0.0088 \pm 0.0012$. This is unexpected from tidal arguments, since the tidal circularisation timescales for many of these systems are considerably shorter than the age of the systems. As a consequence, a lot of theoretical effort has been spent explaining these systems on a case-by-case basis. 


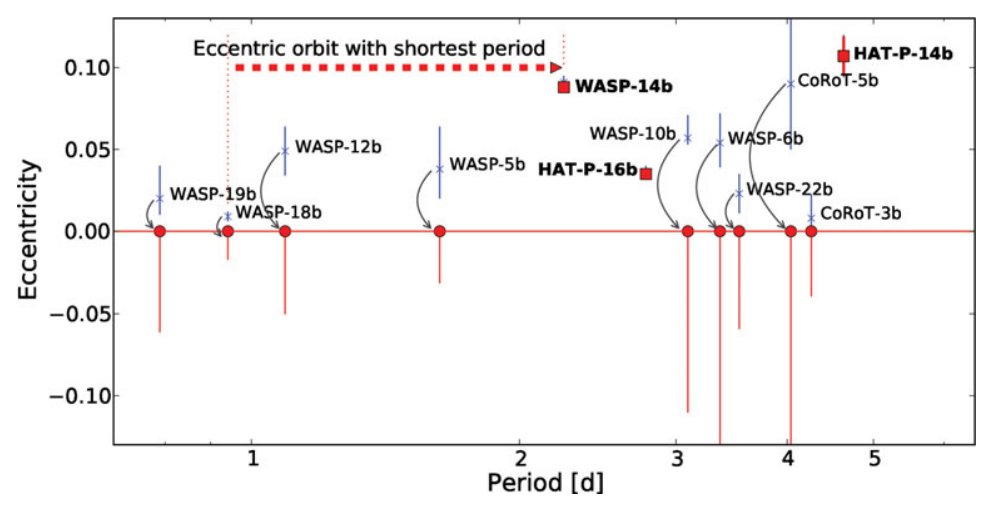

Figure 1. Plot of eccentricity against orbital period. Transiting planets with a reported eccentricity in the Literature $(P<5$ and $e<0.12)$ are represented by crosses. A square (labelled in bold) represents a planet that has a confirmed eccentricity according to our study. Those planets for which we find no evidence for eccentricity are represented by large circles plotted at $e=0$, with the $95 \%$ upper limit on the eccentricity shown on the negative $e$-axis for clarity.

In addition to the combination of radial velocity measurements and transit photometry, one can constrain one projected component of the eccentricity, $e \cos \omega$, where $\omega$ is the argument of periastron, by observing the secondary eclipse as the planet passes behind the parent star. A circular orbit would give a value of $e \cos \omega=0$, i.e. an eclipse phase of $\phi=0.5$. López-Morales et al. (2010) observed WASP-12 in the $z^{\prime}$-band from the ground and obtained a result of $e \cos \omega=0.0156 \pm 0.0035$, corresponding to an eclipse phase $\phi=0.510 \pm 0.002$. This is a 4.5- $\sigma$ departure from a circular orbit, but they later revised their estimate to $e \cos \omega=0.016_{-0.009}^{+0.011}$, in an update to the paper before publication. Campo et al. (2011) observed the secondary eclipse of WASP-12 using Spitzer and derived an eclipse phase of $\phi=0.5012 \pm 0.0006$ (3.6 and $5.8 \mu \mathrm{m})$ and $0.5007 \pm 0.0007$ (4.5 and $8.0 \mathrm{\mu m})$. This suggests that one projected component of the eccentricity is consistent with zero. Using new radial velocity data from SOPHIE, we showed that the WASP-12 system probably had a circular orbit (Husnoo et al. 2010, in Press). We suggested that the original detection could have been caused by instrumental systematics. This can be seen in Figure 2 (left). When a Keplerian orbit is substracted from the radial velocity measurements, the scatter in the residuals is larger than the formal uncertainties in the measurements. When there are few radial velocity measurements, or when most of the measurements are taken on a single night, correlated noise may lead to a spurious detection of eccentricity. In the case of WASP-4 and WASP-5 (Figure 2, right, for WASP5 ), the residuals from substracting a Keplerian orbit from our new data showed a clear signal, which is due to stellar activity (an anti-correlation is observed between the line bisector span and the residuals). If unaccounted for, this signal can inflate the eccentricy to a few percent at the few sigma level. In a similar way, Christian et al. (2009) suggested that WASP-10 was eccentric at $6 \%$ at the $14-\sigma$ level, but recently Maciejewski et al. (2011) reanalysed this system and concluded that the eccentricity detection was related to stellar activity, and if this is taken into account, the data is consistent with a circular orbit.

\section{Analysis}

In this study, we take a new look at the orbital eccentricity of 64 known transiting systems. We add new precise radial velocity data using HARPS and SOPHIE for 12 

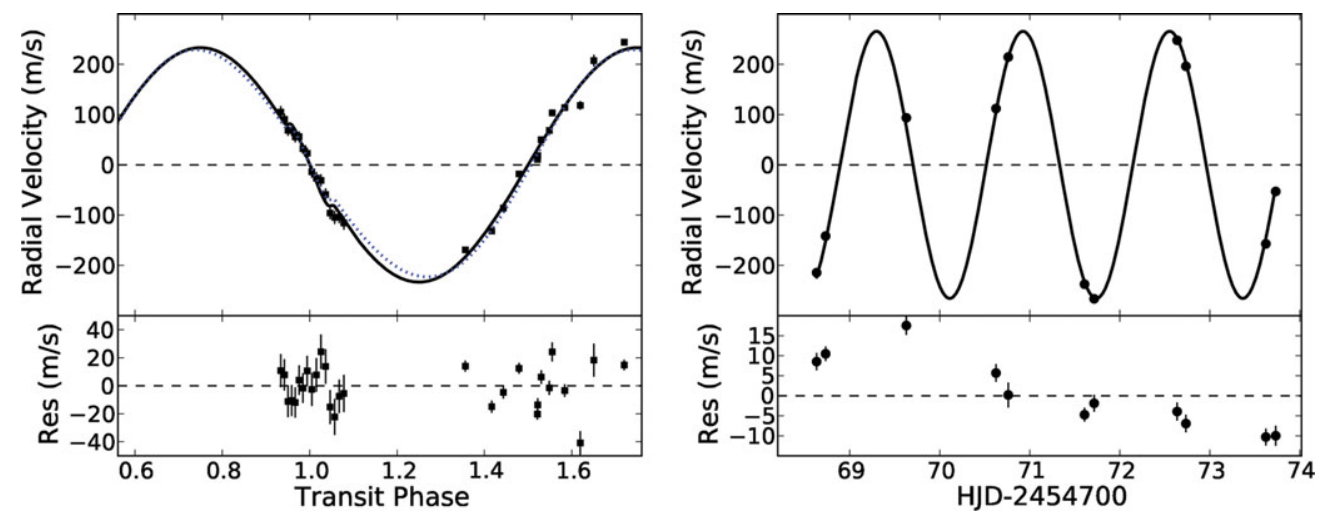

Figure 2. Left: Plot of radial velocity data from SOPHIE against transit phase for WASP-12. An eccentric orbit (dotted line) and a circular orbit (solid line) are overplotted. Note that the scatter of the residuals is larger than the formal uncertainties in the radial velocity measurements. Right: Plot of radial velocity against time for WASP-5. Note the signal in the residuals, due to stellar activity, which could mimic the presence of a second companion in this system.

systems and reanalyse the radial velocity data in the literature for another 42 systems. In addition to these 54 systems, we also include the eccentricities of 8 systems from the literature - HD 189733 and HD 209458 which have well measured eccentricities consistent with zero (see for example Agol et al. (2010) and Laughlin et al. (2005)) and 5 that have precisely measured eccentricities larger than 0.1 (CoRoT-10, HAT-P-15, HD 17156, HD 80606 and XO-3). We also take the eccentricity of the multiplanet system HAT-P-13 from the literature.

For the 54 systems that we analysed, we also collected the relevant orbital constraints from photometry from the original papers. This included the period and mid transit time, but also the secondary eclipse constraint in the form of the eclipse phase $\phi$ or the projected component of the eccentricity, e $\cos \omega$ where available (see Winn et al. 2005, for the relation). For each system in this study (Husnoo et al. 2011, in prep), we include a realistic treatment of the uncertainties, by penalising data taken on the same night in a fashion similar to Pont, Zucker \& Queloz (2006).

Even if all observational uncertainties including correlated noise are taken into account, the value of eccentricity derived from fitting a Keplerian orbit to radial velocity data will always be overestimated. This bias is well known in the literature, and was first pointed out by Lucy \& Sweeney (1971) in relation to short period binaries. The reason is that eccentricity is a positive definite value, and for large observational uncertainties and small eccentricities, any noise will inflate its value. This bias has been studied for exoplanets by Shen \& Turner (2006) and more recently by Zakamska et al. (2010).

\section{Results and conclusion}

Our results are shown in Figure 1 and Figure 3. In Figure 1, we compare the measured eccentricities of 12 transiting systems from the Literature with our own results. We confirm the eccentricities of 3 of these, and find no evidence for eccentricity in the remaining 9. We therefore displace WASP-18b and WASP-12b as the shortest period objects with a confirmed eccentricity, and replace them by WASP-14b and HAT-P-16b.

Our ensemble results are shown in Figure 3, where we have plotted the planetary mass $M_{p}$ against the orbital period $P$. We have deemed systems with precise eccentricities 


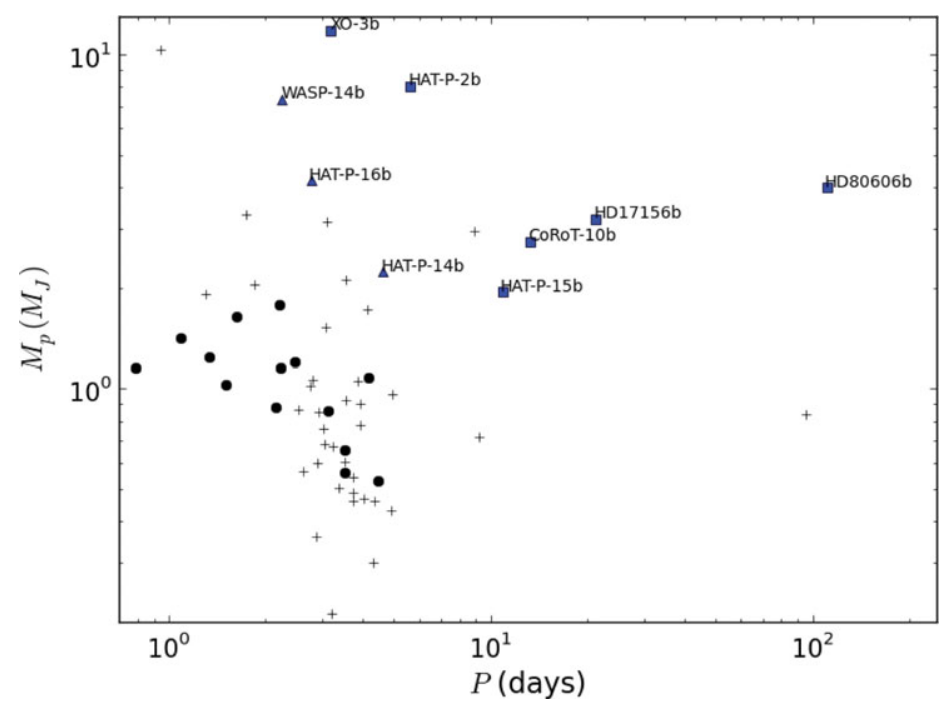

Figure 3. Plot of planetary mass $M_{p}$ against orbital period $P$. Squares denote eccentric systems, and circle denote systems with no evidence for eccentricity. The triangles represent the special case of systems with small but precisely measured eccentricies $(e=3-10 \%$, at the 10- $\sigma$ level or more). The crosses denote systems for which the eccentricity is unknown.

$e>0.1$ as eccentric, and systems with small eccentricities that are not consistent with $e>0.1$, even at the $3-\sigma$ level as compatible circular. An interesting feature in Figure 3 is the mass-period relation. First pointed out by Mazeh et al. (2005), this shows that heavier hot Jupiters tend to orbit at shorter periods. Here, we notice that planets following this trend also have circular orbits, with no confirmed eccentric orbit in this region of parameter space. If one considers tides raised on the planet by the star, light planets at short period are expected to circularise quickly, while heavy planets at longer periods are harder to circularise. We thus expect to see planets on circular orbits on the lower left of Figure 3 while we expect to see planets on eccentric orbits on the upper right. This is indeed what we see in Figure 3, and we consider it to be a signature of tidal evolution.

\section{References}

Agol, E., Cowan, N. B., Knutson, H. A., et al. 2010, ApJ, 721, 1861

Campo, C. J., Harrington, J., Hardy, R. A., et al. 2011, ApJ, 727, id.125

Christian, D. J., Gibson, N. P., Simpson, E. K., et al. 2009, MNRAS, 392, 1585

Hebb, L., Collier-Cameron, A., Loeillet, B., et al. 2009, ApJ, 693, 1920

Hebb, L., Collier-Cameron, A., Triaud, A. H. M. J., et al. 2010, ApJ, 708, 224

Hellier, C., Anderson, D. R., Cameron, A. C., et al. 2009, Nature, 460, 1098

Husnoo, N., et al. 2011a, MNRAS, 413, 2500

Husnoo, N., et al. 2011b, in prep

Laughlin, G., and Marcy, G. W., Vogt, S. S., et al. 2005, ApJL, 629, L121

Lin, D. N. C., Bodenheimer, P., \& Richardson, D. C. 1996, Nature, 380, 606

López-Morales, M., Coughlin, J. L., Sing, D., et al. 2010, ApJL, 716, L36

Lucy, L. B. \& Sweeney, M. A. 1971, AJ, 76, 544

Maciejewski, G., Dimitrov, D., Neuhaeuser, R., et al. 2011, MNRAS, 411, 1204

Mazeh, T., Zucker, S., \& Pont, F. 2005, MNRAS, 356, 955 
Nymeyer, S., Harrington, J., Hardy, R. A., et al. 2010, arXiv:1005.1017

Pont, F., Zucker, S., \& Queloz, D. 2006, MNRAS, 373, 231

Shen, Y. \& Turner, E. L. 2006, ApJ, 685, 553

Triaud, A. H. M. J.., Collier-Cameron, A., \& Queloz, D. 2010, A\& A, 524, A25

Winn, J. N., Noyes, R. W., Holman, M. J., et al. 2005, ApJ, 631, 1215

Zakamska, N. L., Pan, M., \& Ford, E. B. 2010, MNRAS, 1566 